J. Korean Math. Soc. 48 (2011), No. 6, pp. 1203-1223

http://dx.doi.org/10.4134/JKMS.2011.48.6.1203

\title{
OPERATORS SIMILAR TO NORMALOID OPERATORS
}

\author{
Sen Zhu And Chun Guang Li
}

\begin{abstract}
In this paper, the authors investigate the structure of operators similar to normaloid and transloid operators. In particular, we characterize the interior of the set of operators similar to normaloid (transloid, respectively) operators. This gives a concise spectral condition to determine when an operator is similar to a normaloid or transloid operator. Also it is proved that any Hilbert space operator has a compact perturbation with transloid property. This is used to give a negative answer to a problem posed by W. Y. Lee, concerning Weyl's theorem.
\end{abstract}

\section{Introduction}

Throughout this paper, $\mathbb{C}$ and $\mathbb{N}$ denote the set of complex numbers and the set of natural numbers respectively. $\mathscr{H}$ will always denote a complex separable infinite dimensional Hilbert space. We let $\mathscr{B}(\mathscr{H})$ denote the algebra of all bounded linear operators on $\mathscr{H}$, and let $\mathscr{K}(\mathscr{H})$ denote the ideal of compact operators in $\mathscr{B}(\mathscr{H})$.

A well-known result in operator theory asserts that if $T$ is normal on some Hilbert space, then its norm equals its spectral radius. This property plays an important role in the study for the internal structure of normal operators.

Definition 1.1. Let $T \in \mathscr{B}(\mathscr{H})$. Denote by $\gamma(T)$ the spectral radius of $T$, that is, $\gamma(T) \triangleq \max \{|\lambda|: \lambda \in \sigma(T)\}$. We say that $T$ is normaloid if $\gamma(T)=\|T\|$. Let $\mathscr{N}$ denote the set of all normaloid operators on $\mathscr{H}$.

The notion of normaloid operators was first introduced by Wintner [14] in terms of numerical range. An operator $T$ is normaloid if and only if $w(T)=$ $\|T\|$, where $w(T)$ denotes the numerical radius of $T([6])$. Now let us mention some related classes of operators.

Received July 7, 2010

2010 Mathematics Subject Classification. Primary 47A12, 47A58; Secondary 47A10, $47 \mathrm{~A} 25$.

Key words and phrases. normaloidity, transloid operators, similarity, numerical radius, spectral sets.

This research is supported by NNSF of China (10971079,11026038), the Basic Research Foundation of Jilin University(201001001,201103194) and the Young Fund of Department of Mathematics at Jilin University. 
Let $T \in \mathscr{B}(\mathscr{H})$. We say that $T$ is transloid if $\lambda-T$ is normaloid for all $\lambda \in \mathbb{C}$; moreover, if $p(T)$ is normaloid for each polynomial $p(\lambda)$, then we say that $T$ is polynomial-normaloid (p-normaloid for short). Furthermore, if $f(T)$ is normaloid for each rational function $f$ with poles outside $\sigma(T)$, then $T$ is called a von Neumann operator. We let $\mathscr{T}, \mathscr{P}$ and $\mathscr{V}$ denote the set of all transloid operators, the set of all p-normaloid operators and the set of all von Neumann operators on $\mathscr{H}$, respectively. It is obvious that $\mathscr{V} \subset \mathscr{P} \subset \mathscr{T} \subset \mathscr{N}$.

The concept of a von Neumann operator is closely related to a notion of spectral sets due to von Neumann [13]. If $T \in \mathscr{B}(\mathscr{H})$ and $\sigma$ is a compact subset of $\mathbb{C}$, then $\sigma$ is called a spectral set for $T$ if $\sigma(T) \subset \sigma$ and, for each rational function $f$ with poles outside $\sigma$,

$$
\|f(T)\| \leq \max \{|f(\lambda)|: \lambda \in \sigma\} .
$$

By the spectral mapping theorem, it is easily seen that $T$ is a von Neumann operator if and only if $\sigma(T)$ is a spectral set for $T$ ([4, Theorem 9.7]).

It is well known that each subnormal operator is a von Neumann operator. Herrero [7] extended a classical result of Rota [12] concerning an operator model. As a corollary, Herrero obtained the following result.

Theorem $1.2([8]$, Corollary 3.35). Given $T \in \mathscr{B}(\mathscr{H})$ and a bounded neighborhood $\Phi$ of $\sigma(T), \bar{\Phi}$ is a spectral set for some $T^{\prime}$ similar to $T$.

Gilfeather [5] used Rota's original construction to give a different proof of Theorem 1.2. This result shows that, for any fixed neighborhood $\Phi$ of $\sigma(T)$, no matter what size it is, there exists an operator similar to $T$ admitting $\bar{\Phi}$ as a spectral set. Then it is natural to ask: given an operator $T \in \mathscr{B}(\mathscr{H})$, does there exist an operator, similar to $T$, admitting $\sigma(T)$ as a spectral set? In other words, is there a von Neumann operator similar to $T$ ?

Recall that two operators $A, B \in \mathscr{B}(\mathscr{H})$ are similar (denoted by $A \sim B$ ) if there exists an invertible operator $X \in \mathscr{B}(\mathscr{H})$ such that $A X=X B$. Given $T \in \mathscr{B}(\mathscr{H})$, the similarity orbit $\mathcal{S}(T)$ of $T$ is the set $\{X \in \mathscr{B}(\mathscr{H}): X \sim T\}$. Given a subset $\mathscr{E}$ of $\mathscr{B}(\mathscr{H})$, denote $\mathcal{S}(\mathscr{E})=\cup_{X \in \mathscr{E}} \mathcal{S}(X)$. We call $\mathcal{S}(\mathscr{E})$ the similarity orbit of $\mathscr{E}$. Thus our former question is: $\mathcal{S}(\mathscr{V})=\mathscr{B}(\mathscr{H})$ ?

It is easy to see that each nonzero quasinilpotent operator is not similar to any von Neumann operator. So $\mathcal{S}(\mathscr{V}) \neq \mathscr{B}(\mathscr{H})$. What are the interior and the closure of $\mathcal{S}(\mathscr{V})$ ? Also we can consider similar questions for the larger classes $\mathscr{N}, \mathscr{T}$ and $\mathscr{P}$.

In [2], Apostol and Morrel introduced a class of operators called simple models, which are dense in $\mathscr{B}(\mathscr{H})$. Each simple model is constructed by some subnormal operators. It is not difficult to see that each simple model is similar to a direct sum of subnormal operators and their adjoints. Hence each simple model is similar to a von Neumann operator. Then one can conclude that $\overline{\mathcal{S}(\mathscr{V})}=\mathscr{B}(\mathscr{H})$. So $\overline{\mathcal{S}(\mathscr{N})}=\overline{\mathcal{S}(\mathscr{T})}=\overline{\mathcal{S}(\mathscr{P})}=\mathscr{B}(\mathscr{H})$. 
The purpose of this paper is to investigate the stability of normaloidity and the above-mentioned properties under similarity and small perturbations. More precisely, this paper is devoted to solving the following problems:

1. Characterize the operators $T$ satisfying $\mathcal{S}(T) \subset \mathscr{N}, \mathcal{S}(T) \subset \mathscr{T}, \mathcal{S}(T) \subset$ $\mathscr{P}$ and $\mathcal{S}(T) \subset \mathscr{V}$, respectively.

2. Characterize the interiors of $\mathcal{S}(\mathscr{N}), \mathcal{S}(\mathscr{T}), \mathcal{S}(\mathscr{P})$ and $\mathcal{S}(\mathscr{V})$, respectively.

In this paper, we completely solve the above problems. As we shall see later, the answers to Problem 2 enable us to obtain a deep understanding of the internal structure of normaloid and transloid operators.

\section{Notations and results}

In order to state our results we first recall some notations and terminologies.

Let $\sigma$ be a subset of $\mathbb{C}$. We denote by iso $\sigma$ the set of all isolated points of $\sigma$. Let conv $\sigma$ denote the convex hull of $\sigma$, namely the intersection of all convex sets containing $\sigma$. The interior of $\sigma$ is denoted by $\sigma^{\circ}$. Let card $\sigma$ denote the cardinal number of $\sigma$.

Let $T \in \mathscr{B}(\mathscr{H})$. We denote by $\sigma(T)$ and $\sigma_{p}(T)$ the spectrum of $T$ and the point spectrum of $T$ respectively. Denote by $\operatorname{ker} T$ and $\operatorname{ran} T$ the kernel of $T$ and the range of $T$ respectively. $T$ is called a semi-Fredholm operator, if $\operatorname{ran} T$ is closed and either nul $T$ or nul $T^{*}$ is finite, where nul $T \triangleq \operatorname{dim} \operatorname{ker} T$ and nul $T^{*} \triangleq \operatorname{dim} \operatorname{ker} T^{*}$; in this case, ind $T \triangleq \operatorname{nul} T-$ nul $T^{*}$ is called the index of $T$. In particular, if $-\infty<$ ind $T<\infty$, then $T$ is called a Fredholm operator. The Wolf spectrum $\sigma_{\text {lre }}(T)$ and the essential spectrum $\sigma_{e}(T)$ are defined by

$$
\sigma_{\text {lre }}(T) \triangleq\{\lambda \in \mathbb{C}: T-\lambda \text { is not semi-Fredholm }\},
$$

and

$$
\sigma_{e}(T) \triangleq\{\lambda \in \mathbb{C}: T-\lambda \text { is not Fredholm }\},
$$

respectively. $\rho_{s-F}(T) \triangleq \mathbb{C} \backslash \sigma_{\text {lre }}(T)$ is called the semi-Fredholm domain of $T$.

For $\sigma$, a clopen subset of $\sigma(T), E(\sigma ; T)$ denotes the corresponding Riesz idempotent and $\mathscr{H}(\sigma ; T) \triangleq$ ran $E(\sigma ; T)$, the corresponding Riesz spectral space. Write $\mathscr{H}(\lambda ; T)$ for $\mathscr{H}(\{\lambda\} ; T)$. If $\operatorname{dim} \mathscr{H}(\lambda ; T)<\infty$, then $\lambda$ is called a normal eigenvalue of $T$. The set of all normal eigenvalues of $T$ is denoted by $\sigma_{0}(T)$. Denote $\sigma_{1}(T)=\{\lambda \in$ iso $\sigma(T): \operatorname{dim} \mathscr{H}(\lambda ; T)=1\}$. It is obvious that $\sigma_{1}(T) \subset \sigma_{0}(T)$. Given a subset $\mathscr{E}$ of $\mathscr{B}(\mathscr{H})$, let $\overline{\mathscr{E}}$ and $\mathscr{E} \circ$ denote the norm-closure and the interior of $\mathscr{E}$ respectively.

The main results of this paper are listed as follows.

Theorem 2.1. Let $T \in \mathscr{B}(\mathscr{H})$. Then the following are equivalent:

(i) $\mathcal{S}(T) \subseteq \mathscr{N}$

(ii) $\mathcal{S}(T) \subseteq \mathscr{T}$;

(iii) $\mathcal{S}(T) \subseteq \mathscr{P}$;

(iv) $\mathcal{S}(T) \subseteq \mathscr{V}$; 
(v) $T=\lambda I$ for some $\lambda \in \mathbb{C}$.

Theorem 2.2. Given $T \in \mathscr{B}(\mathscr{H})$ and $\varepsilon>0$, there exists $K \in \mathscr{K}(\mathscr{H})$ with $\|K\|<\varepsilon$ such that $T+K \notin \mathcal{S}(\mathscr{P})$.

Corollary 2.3. $\mathcal{S}(\mathscr{P})$ and $\mathcal{S}(\mathscr{V})$ are nowhere dense in $\mathscr{B}(\mathscr{H})$.

Theorem 2.4 (Main Theorem 1). Let $T \in \mathscr{B}(\mathscr{H})$. Then $T \in \mathcal{S}(\mathscr{N})^{\circ}$ if and only if each $\lambda \in \sigma(T)$ with $|\lambda|=\gamma(T)$ satisfies that $\lambda \in$ iso $\sigma(T)$ and $\operatorname{dim} \mathscr{H}(\lambda ; T)=1$.

Theorem 2.5 (Main Theorem 2). Let $T \in \mathscr{B}(\mathscr{H})$. Then the following are equivalent:

(i) $T \in \mathcal{S}(\mathscr{T})^{\circ}$;

(ii) $\left[\sigma(T) \backslash \sigma_{1}(T)\right] \subset\left[\operatorname{conv} \sigma_{1}(T)\right]^{\circ}$;

(iii) there exists a finite subset $\Gamma$ of $\sigma_{1}(T)$ such that $[\sigma(T) \backslash \Gamma] \subset[\operatorname{conv} \Gamma]^{\circ}$.

Theorem 2.6 (Main Theorem 3). $\mathscr{T}+\mathscr{K}(\mathscr{H})=\mathscr{B}(\mathscr{H})=\mathscr{N}+\mathscr{K}(\mathscr{H})$.

Theorem 2.7. Given $T \in \mathscr{B}(\mathscr{H})$ and $\varepsilon>0$, there exists $K \in \mathscr{K}(\mathscr{H})$ with $\|K\|<\varepsilon$ such that $T+K \in \mathcal{S}(\mathscr{T})^{\circ}$.

Corollary 2.8. $\overline{\mathcal{S}(\mathscr{T})^{\circ}}=\mathscr{B}(\mathscr{H})=\overline{\mathcal{S}(\mathscr{N})^{\circ}}$.

The rest part of this paper is organized as follows. In Section 3, we give the proof of Theorem 2.1. Section 4 is devoted to the proof of Theorem 2.2. In Section 5, we shall prove Theorem 2.4. The proofs of Theorems 2.5, 2.6 shall be provided in Section 6. In Section 7, we give the proof of Theorem 2.7.

\section{Proof of Theorem 2.1}

Proposition 3.1. Let $T \in \mathscr{B}(\mathscr{H})$ and suppose that $T$ admits a nontrivial invariant subspace $M$, that is, $T(M) \subset M$ and $\{0\} \neq M \neq \mathscr{H}$. If $M$ is not a reducing subspace of $T$, then $\mathcal{S}(T)$ is not a subset of $\mathscr{N}$.

Proof. By hypothesis, we may assume that $T$ admits the following matrix representation

$$
T=\left[\begin{array}{cc}
A & C \\
0 & B
\end{array}\right] \begin{gathered}
M \\
M^{\perp}
\end{gathered}
$$

where $C \neq 0$ and $M^{\perp}$ denotes the orthogonal complement of $M$. Obviously we can choose some $n \in \mathbb{N}$ such that $n\|C\| \geq\|A\|+\|B\|+1$. Put

$$
W=\left[\begin{array}{cc}
n I_{1} & 0 \\
0 & I_{2}
\end{array}\right] \begin{gathered}
M \\
M^{\perp},
\end{gathered}
$$

where $I_{1}$ is the unit operator on $M$ and $I_{2}$ is the unit operator on $M^{\perp}$. It is obvious that $W$ is invertible. Denote $T^{\prime}=W T W^{-1}$. Then $T^{\prime} \sim T$ and

$$
T^{\prime}=\left[\begin{array}{cc}
A & n C \\
0 & B
\end{array}\right] .
$$

One can easily check that $\left\|T^{\prime}\right\| \geq n\|C\|>\|A\|+\|B\| \geq \gamma\left(T^{\prime}\right)$. Hence $T^{\prime} \notin \mathscr{N}$ and $\mathcal{S}(T)$ is not a subset of $\mathscr{N}$. 

ted.

The following lemma is a consequence of Theorem 1.2 and its proof is omit-

Lemma 3.2. Let $T \in \mathscr{B}(\mathscr{H})$. Then, given $\varepsilon>0$, there exists $T^{\prime} \in \mathscr{B}(\mathscr{H})$ such that $T^{\prime} \sim T$ and $\left\|T^{\prime}\right\|<\gamma\left(T^{\prime}\right)+\varepsilon$.

Lemma 3.3 ([8], Lemma 8.1). Let $Q \in \mathscr{B}(\mathscr{H})$ be quasinilpotent. If $Q^{k} \neq 0$ for all $k \geq 1$, then $\overline{\mathcal{S}(Q)}$ contains all compact quasinilpotent operators on $\mathscr{H}$.

Lemma 3.4 ([1], Theorem 9.1). Let $T \in \mathscr{B}(\mathscr{H})$ and suppose that $\sigma_{\text {lre }}(T)$ has limit points. If $A \in \mathscr{B}(\mathscr{H})$ and $\sigma(T) \subset \sigma(A)=\sigma_{\text {lre }}(A)$, then $A \in \overline{\mathcal{S}(T)}$.

Lemma 3.5 ([11], Theorem 2.10). Let $T \in \mathscr{B}(\mathscr{H})$ and suppose that $\sigma(T)=$ $\sigma_{1} \cup \sigma_{2}$, where $\sigma_{i}(i=1,2)$ are clopen subsets of $\sigma(T)$ and $\sigma_{1} \cap \sigma_{2}=\emptyset$. Then $\mathscr{H}\left(\sigma_{1} ; T\right)+\mathscr{H}\left(\sigma_{2} ; T\right)=\mathscr{H}, \mathscr{H}\left(\sigma_{1} ; T\right) \cap \mathscr{H}\left(\sigma_{2} ; T\right)=\{0\}$ and $T$ admits the following matrix representation

$$
T=\left[\begin{array}{cc}
T_{1} & 0 \\
0 & T_{2}
\end{array}\right] \begin{aligned}
& \mathscr{H}\left(\sigma_{1} ; T\right) \\
& \mathscr{H}\left(\sigma_{2} ; T\right)
\end{aligned}
$$

where $\sigma\left(T_{i}\right)=\sigma_{i}(i=1,2)$.

Lemma 3.6 ([8], Corollary 3.22). Let $T \in \mathscr{B}(\mathscr{H})$ and suppose that $T$ admits the following representation

$$
T=\left[\begin{array}{cc}
A & C \\
0 & B
\end{array}\right] \mathscr{H}_{1}
$$

where $\sigma(A) \cap \sigma(B)=\emptyset$. Then $T \sim A \oplus B$.

Using the above lemma, we can obtain the following lemma whose proof is left to the reader.

Corollary 3.7. Let $T \in \mathscr{B}(\mathscr{H})$ and suppose that $\sigma$ is a clopen subset of $\sigma(T)$. Then

$$
T=\left[\begin{array}{cc}
A & * \\
0 & B
\end{array}\right] \quad \begin{gathered}
\mathscr{H}(\sigma ; T) \\
\mathscr{H}(\sigma ; T)^{\perp}
\end{gathered}\left[\begin{array}{cc}
A & 0 \\
0 & B
\end{array}\right] \begin{aligned}
& \mathscr{H}(\sigma ; T) \\
& \mathscr{H}(\sigma ; T)^{\perp}
\end{aligned}
$$

where $\sigma(A)=\sigma$ and $\sigma(B)=\sigma(T) \backslash \sigma$.

Proof of Theorem 2.1. Since $\mathscr{V} \subset \mathscr{P} \subset \mathscr{T} \subset \mathscr{N}$, the following relations are obvious:

$$
\text { "(v) } \Rightarrow \text { (iv) } \Rightarrow \text { (iii) } \Rightarrow \text { (ii) } \Rightarrow \text { (i)". }
$$

Thus it suffices to prove that "(i) $\Rightarrow(\mathrm{v})$ ".

Now we assume that $\mathcal{S}(T) \subset \mathscr{N}$. We shall prove (v) by four steps.

Step 1. $\sigma(T)$ is connected. Otherwise, we may assume that $\sigma(T)=\sigma_{1} \cup \sigma_{2}$, where $\sigma_{1}, \sigma_{2}$ are two nonempty clopen subsets of $\sigma(T)$ and $\sigma_{1} \cap \sigma_{2}=\emptyset$. By Corollary 3.7, $T$ can be written as

$$
T=\left[\begin{array}{cc}
A & C \\
0 & B
\end{array}\right] \mathscr{H}_{2}
$$


where $\sigma(A)=\sigma_{1}$ and $\sigma(B)=\sigma_{2}$. By Lemma 3.6, we deduce that

$$
T \sim\left[\begin{array}{cc}
A & 0 \\
0 & B
\end{array}\right] \sim\left[\begin{array}{cc}
A & R \\
0 & B
\end{array}\right]
$$

for every operator $R$ mapping $\mathscr{H}_{2}$ into $\mathscr{H}_{1}$. Now we choose an operator $R_{0}$ mapping $\mathscr{H}_{2}$ into $\mathscr{H}_{1}$ with $\left\|R_{0}\right\|>\gamma(T)$. Put

$$
T^{\prime}=\left[\begin{array}{cc}
A & R_{0} \\
0 & B
\end{array}\right] .
$$

Then $T^{\prime} \sim T$ and hence $\gamma\left(T^{\prime}\right)=\gamma(T)<\left\|R_{0}\right\| \leq\left\|T^{\prime}\right\|, T^{\prime} \notin \mathscr{N}$. Thus we find an operator $T^{\prime}$ similar to $T$ such that $T^{\prime} \notin \mathscr{N}$, a contradiction.

Step 2. $\sigma(T)$ is a singleton. For a proof by contrary, we assume that $\sigma(T)$ is not a singleton. Since we have proved that $\sigma(T)$ is connected, it follows that iso $\sigma(T)=\emptyset$. Then each $\lambda_{0} \in \partial \sigma(T)$ is a limit point of $\sigma(T)$ and $\partial \sigma(T) \subset$ $\sigma_{\text {lre }}(T)$. Hence $\sigma_{\text {lre }}(T)$ has limit points.

Obviously we can choose an operator $X \in \mathscr{B}(\mathscr{H})$ such that $\sigma(X)=\sigma_{\text {lre }}(X)$ $=\{\lambda \in \mathbb{C}:|\lambda| \leq\|T\|+1\}$. Then $\|X\| \geq\|T\|+1$. It follows from Lemma 3.4 that $X \in \overline{\mathcal{S}(T)}$, then there exists $T^{\prime} \in \mathcal{S}(T)$ such that $\left\|X-T^{\prime}\right\|<1$. A direct computation shows that

$$
\begin{aligned}
\left\|T^{\prime}\right\| & =\left\|X-\left(X-T^{\prime}\right)\right\| \geq\|X\|-\left\|X-T^{\prime}\right\| \\
& >\|T\|+1-1=\|T\| \geq \gamma(T)=\gamma\left(T^{\prime}\right) .
\end{aligned}
$$

Hence $T^{\prime} \notin \mathscr{N}$ and $\mathcal{S}(T)$ is not a subset of $\mathscr{N}$, a contradiction. Therefore we may assume without loss of generality that $\sigma(T)=\left\{\lambda_{0}\right\}$.

Step 3. $T-\lambda_{0}$ is nilpotent. Otherwise, $\left(T-\lambda_{0}\right)^{k} \neq 0$ for all $k \geq 1$. Then, by Lemma $3.3, \overline{\mathcal{S}\left(T-\lambda_{0}\right)}=\overline{\mathcal{S}(T)}-\lambda_{0}$ contains all compact quasinilpotent operators. Arbitrarily choose a finite-rank operator $F \in \mathscr{B}(\mathscr{H})$ such that $\sigma(F)=\{0\}$ and $\|F\|>\left|\lambda_{0}\right|+1$. Then $F \in \overline{\mathcal{S}\left(T-\lambda_{0}\right)}$ and hence there exists $T^{\prime} \in \mathcal{S}\left(T-\lambda_{0}\right)$ such that $\left\|F-T^{\prime}\right\|<1$. Using a similar argument as in Step 2, one can prove that $\gamma\left(T^{\prime}\right)<\left\|T^{\prime}\right\|$. Hence $\mathcal{S}(T)$ is not a subset of $\mathscr{N}$, a contradiction. Therefore we may assume without loss of generality that $\left(T-\lambda_{0}\right)^{k}=0$ for some $k \geq 1$.

Step 4. $T=\lambda_{0} I$. If not, then $T-\lambda_{0} \neq 0$. Denote $M=\operatorname{ker}\left(T-\lambda_{0}\right)$. Then $\mathscr{H} \neq M \neq\{0\}$. In this case, $T-\lambda_{0}$ admits the following representation

$$
T-\lambda_{0}=\left[\begin{array}{ll}
0 & C \\
0 & B
\end{array}\right] \begin{gathered}
M \\
M^{\perp} .
\end{gathered}
$$

Obviously $C \neq 0$. Then $M$ does not reduce $T$. According to Proposition 3.1, we can deduce that $\mathcal{S}(T)$ is not a subset of $\mathscr{N}$, a contradiction. Therefore we conclude that $T=\lambda_{0} I$.

Corollary 3.8. Let $T \in \mathscr{B}(\mathscr{H})$. Then each $X$ in $\mathcal{S}(T)$ is normal if and only if $T=\lambda I$ for some $\lambda \in \mathbb{C}$. 


\section{Proof of Theorem 2.2}

Proposition 4.1. $\mathscr{N}$ is a closed subset of $\mathscr{B}(\mathscr{H})$.

Proof. Let $\left\{T_{n}\right\}_{n=0}^{\infty}$ be a sequence of operators on $\mathscr{H}$. Assume that $T_{n} \in \mathscr{N}$ for all $n \geq 1$ and $\left\|T_{n}-T\right\| \rightarrow 0(n \rightarrow \infty)$. It suffices to prove that $\|T\| \leq \gamma(T)$.

Since $\left\|T_{n}-T\right\| \rightarrow 0(n \rightarrow \infty)$, we have $\lim _{n \rightarrow \infty}\left\|T_{n}\right\|=\|T\|$ and, by the upper semi-continuity of spectrum, $\lim \sup _{n \rightarrow \infty} \gamma\left(T_{n}\right) \leq \gamma(T)$. $T_{n} \in \mathscr{N}$ implies that $\left\|T_{n}\right\|=\gamma\left(T_{n}\right)$ for all $n \geq 1$. Thus we obtain $\|T\| \leq \gamma(T)$.

The following two corollaries are direct consequences of Proposition 4.1.

Corollary 4.2. $\mathscr{T}, \mathscr{P}$ and $\mathscr{V}$ are closed subsets of $\mathscr{B}(\mathscr{H})$.

Corollary 4.3. An operator $T \in \mathscr{B}(\mathscr{H})$ is a von Neumann operator if and only if $f(T)$ is normaloid for all $f \in \operatorname{Hol}(\sigma(T))$.

Here and in what follows, we let $\operatorname{Hol}(\sigma(T))$ denote the set of all functions $f$ which are analytic on some neighborhood of $\sigma(A)$ (the neighborhood depends on $f$ ).

Lemma 4.4 ([8], Proposition 4.29). Let $T \in \mathscr{B}(\mathscr{H})$ and suppose that $\emptyset \neq \Gamma \subset$ $\sigma_{\text {lre }}(T)$. Then, given $\varepsilon>0$, there exists a compact operator $K$ with $\|K\|<\varepsilon$ such that

where

$$
T+K=\left[\begin{array}{cc}
N & * \\
0 & A
\end{array}\right] \begin{gathered}
M \\
M^{\perp}
\end{gathered}
$$

(i) $N$ is a diagonal normal operator of uniformly infinite multiplicity, $\sigma(N)=\sigma_{\text {lre }}(N)=\bar{\Gamma}$,

(ii) $\sigma(T)=\sigma(A), \sigma_{1}(T)=\sigma_{1}(A)$, and

(iii) $\sigma_{\text {lre }}(T)=\sigma_{\text {lre }}(A)$, ind $(T-\lambda)=$ ind $(A-\lambda)$ for all $\lambda \in \rho_{s-F}(T)$.

Lemma 4.5 ([3], Proposition 6.9). Let $T \in \mathscr{B}(\mathscr{H})$. Then $\partial \sigma(T) \subset\left[\sigma_{\text {lre }}(T) \cup\right.$ $\left.\sigma_{0}(T)\right]$.

The following result means that $\mathscr{N}$ is nowhere dense in $\mathscr{B}(\mathscr{H})$.

Theorem 4.6. Given $T \in \mathscr{B}(\mathscr{H})$ and $\varepsilon>0$, there exists $K \in \mathscr{K}(\mathscr{H})$ with $\|K\|<\varepsilon$ such that $T+K \notin \mathscr{N}$.

Proof. First we can choose a $\lambda_{0} \in \partial \sigma(T)$ satisfying that $\left|\lambda_{0}\right|=\gamma(T)$. Then it can be seen from Lemma 4.5 that either $\lambda_{0} \in \sigma_{p}(T)$ or $\lambda_{0} \in \sigma_{\text {lre }}(T)$.

Case 1. $\lambda_{0} \in \sigma_{p}(T)$. In this case, there exists a unit vector $e \in \mathscr{H}$ such that $T e=\lambda_{0} e$. Arbitrarily choose an $\operatorname{ONB}\left\{e_{n}\right\}_{n=1}^{\infty}$ such that $e_{1}=e$. Thus $T$ can be written as

$$
T=\left[\begin{array}{cccc}
\lambda_{0} & * & * & \cdots \\
0 & * & * & \cdots \\
0 & * & * & \cdots \\
\vdots & \vdots & \vdots & \ddots
\end{array}\right] \begin{gathered}
e_{1} \\
e_{2} \\
e_{3}
\end{gathered} \vdots\left[\begin{array}{cc}
\lambda_{0} & C \\
0 & A
\end{array}\right]\left\{e_{1}^{e_{1}}\right\}^{\perp}
$$


Then $\gamma(A) \leq \gamma(T)$. It is obvious that we can choose a $K \in \mathscr{K}(\mathscr{H})$ with $\|K\|<\varepsilon$ such that

$$
T+K=\left[\begin{array}{cc}
\lambda_{0} & \bar{C} \\
0 & A
\end{array}\right]\left\{e_{1}\right\}^{\perp}=\left[\begin{array}{cccc}
\lambda_{0} & \lambda_{1} & \lambda_{2} & \cdots \\
0 & * & * & \cdots \\
0 & * & * & \cdots \\
\vdots & \vdots & \vdots & \ddots
\end{array}\right] e_{1}, e_{2},
$$

where $\lambda_{i} \neq 0$ for all $i \geq 1$. Then

$$
\|T+K\|^{2} \geq \sum_{i=0}^{\infty}\left|\lambda_{i}\right|^{2}>\left|\lambda_{0}\right|^{2}=\gamma(T)^{2}=\gamma(T+K)^{2} .
$$

Hence we conclude that $T+K \notin \mathscr{N}$.

Case 2. $\lambda_{0} \in \sigma_{\text {lre }}(T)$. By Lemma 4.4, there exists $K_{1} \in \mathscr{K}(\mathscr{H})$ with $\left\|K_{1}\right\|<\varepsilon / 2$ such that

$$
T+K_{1}=\left[\begin{array}{cc}
\lambda_{0} & C \\
0 & A
\end{array}\right] \underset{\{f\}^{\perp}}{f}
$$

where $\sigma(A)=\sigma(T)$. Hence $\gamma\left(T+K_{1}\right)=\gamma(T)$ and $\lambda_{0} \in \partial \sigma(A)$. It is obvious that $\lambda_{0} \in \sigma_{p}\left(T+K_{1}\right)$ and $f \in \operatorname{ker}\left(\lambda_{0}-T\right)$. Using a similar argument as in Case 1 , we can find a $K_{2} \in \mathscr{K}(\mathscr{H})$ with $\left\|K_{2}\right\|<\varepsilon / 2$ such that $T+K_{1}+K_{2} \notin \mathscr{N}$. Set $K=K_{1}+K_{2}$. Then $K$ satisfies all requirements.

The following result can be inferred from the proof of Theorem 4.6.

Corollary 4.7. Let $T \in \mathscr{B}(\mathscr{H})$. Suppose that $\lambda_{0} \in \sigma(T)$ and $\left|\lambda_{0}\right|=\gamma(T)$. Then, given $\varepsilon>0$, there exists $K \in \mathscr{K}(\mathscr{H})$ with $\|K\|<\varepsilon$ such that

$$
T+K=\left[\begin{array}{cc}
\lambda_{0} & * \\
0 & A
\end{array}\right]\left\{\begin{array}{c}
e \\
e\}^{\perp}
\end{array}\right.
$$

where $e \in \mathscr{H}$ is a unit vector and $\sigma(A) \subset \sigma(T)$.

Proof of Theorem 2.2. Arbitrarily choose a $\lambda_{0} \in \partial \sigma_{e}(T)$ satisfying that $\left|\lambda_{0}\right|=$ $\max \left\{|\lambda|: \lambda \in \sigma_{e}(T)\right\}$. It is obvious that $\lambda_{0} \in \partial \sigma(T) \cap \sigma_{\text {lre }}(T)$. Also one can note that $\lambda_{0}$ is in the boundary of the unbounded component of $\mathbb{C} \backslash \sigma(T)$.

For given $\varepsilon>0$, it follows from Lemma 4.4 that there exists $K_{1} \in \mathscr{K}(\mathscr{H})$ with $\left\|K_{1}\right\|<\varepsilon / 2$ such that

$$
T+K_{1}=\left[\begin{array}{ccc}
\lambda_{0} & \mu_{0} & C \\
0 & \lambda_{0} & B \\
0 & 0 & A
\end{array}\right] \begin{gathered}
e \\
f \\
\{e, f\}^{\perp}
\end{gathered},
$$

where $e, f \in \mathscr{H}$ are two orthogonal normalized vectors and $\sigma(A)=\sigma(T)$. Obviously, since $\lambda_{0} \in \partial \sigma(T)$, we can choose $\lambda_{1}$ in the unbounded component of $\mathbb{C} \backslash \sigma(T)$ and $\mu_{1} \neq 0$ such that $\left|\lambda_{0}-\lambda_{1}\right|+\left|\mu_{0}-\mu_{1}\right|<\varepsilon / 2$. Set

$$
K_{2}=\left[\begin{array}{ccc}
\lambda_{1}-\lambda_{0} & \mu_{1}-\mu_{0} & 0 \\
0 & \lambda_{1}-\lambda_{0} & 0 \\
0 & 0 & 0
\end{array}\right] \underset{\{e, f\}^{\perp}}{\underset{f}{f}}, K=K_{1}+K_{2} .
$$


Then $K \in \mathscr{K}(\mathscr{H}),\|K\|<\varepsilon$ and

$$
T+K=\left[\begin{array}{ccc}
\lambda_{1} & \mu_{1} & C \\
0 & \lambda_{1} & B \\
0 & 0 & A
\end{array}\right]\left\{\begin{array}{c}
e \\
f \\
\end{array}\right.
$$

One can verify that $\sigma(T+K)=\left\{\lambda_{1}\right\} \cup \sigma(A)$ and $\lambda_{1} \in \sigma_{0}(T+K)$. To complete the proof, it suffices to prove that $T+K \notin \mathcal{S}(\mathscr{P})$.

For a proof by contrary, we assume that $T^{\prime} \in \mathscr{P}$ and $T^{\prime} \sim T+K$. Then $\sigma\left(T^{\prime}\right)=\sigma(T+K)$, nul $\left(T^{\prime}-\lambda_{1}\right)=\operatorname{nul}\left(T+K-\lambda_{1}\right)=1$ and nul $\left(T^{\prime}-\right.$ $\left.\lambda_{1}\right)^{2}=\operatorname{nul}\left(T+K-\lambda_{1}\right)^{2}=2$. Therefore, with respect to some suitably chosen ONB $\{g, h\}$ of $\operatorname{ker}\left(T^{\prime}-\lambda_{1}\right)^{2}, T^{\prime}$ can be written as

$$
T^{\prime}=\left[\begin{array}{ccc}
\lambda_{1} & \mu_{2} & C_{1} \\
0 & \lambda_{1} & B_{1} \\
0 & 0 & A_{1}
\end{array}\right] \begin{gathered}
g \\
h \\
\{g, h\}^{\perp}
\end{gathered},
$$

where $\mu_{2} \neq 0$.

Note that $\lambda_{1}$ is an isolated point of $\sigma\left(T^{\prime}\right)$ and $\lambda_{1}$ is in the unbounded component of $\mathbb{C} \backslash\left[\sigma\left(T^{\prime}\right) \backslash\left\{\lambda_{1}\right\}\right]$, then we can define a function $f \in \operatorname{Hol}(\sigma(T))$ such that $f(\lambda)=\lambda$ on a disk $\Omega_{1}$ centered at $\lambda_{1}$ and $f(\lambda) \equiv 0$ on a simply-connected neighborhood $\Omega_{2}$ of $\sigma(T) \backslash\left\{\lambda_{1}\right\}$, where $\overline{\Omega_{1}} \cap \overline{\Omega_{2}}=\emptyset$. One can deduce that

$$
f\left(T^{\prime}\right)=\left[\begin{array}{ccc}
\lambda_{1} & \mu_{2} & * \\
0 & \lambda_{1} & * \\
0 & 0 & 0
\end{array}\right] \begin{gathered}
g \\
h \\
\{g, h\}^{\perp}
\end{gathered} .
$$

It is obvious that $\gamma\left(f\left(T^{\prime}\right)\right)=\left|\lambda_{1}\right|<\sqrt{\left|\lambda_{1}\right|^{2}+\left|\mu_{2}\right|^{2}} \leq\left\|f\left(T^{\prime}\right)\right\|$ and hence $f\left(T^{\prime}\right) \notin \mathscr{N}$.

On the other hand, by Runge's approximation theorem, there exists polynomials $\left\{p_{n}(\lambda)\right\}_{n=1}^{\infty}$ such that $\sup \left\{\left|p_{n}(\lambda)-f(\lambda)\right|: \lambda \in \Omega_{1} \cup \Omega_{2}\right\} \rightarrow 0$. Hence $\left\|p_{n}\left(T^{\prime}\right)-f\left(T^{\prime}\right)\right\| \rightarrow 0$. Since $T^{\prime} \in \mathscr{P}$, it follows that $p_{n}\left(T^{\prime}\right) \in \mathscr{N}$ and, by Proposition 4.1, $f\left(T^{\prime}\right) \in \mathscr{N}$, a contradiction. Thus we complete the proof.

\section{Proof of Theorem 2.4}

Before we give the proof of Theorem 2.4, let us make some preparations.

Proposition 5.1. Let $T \in \mathscr{B}(\mathscr{H})$. Suppose that $\lambda_{0} \in \sigma(T)$ and $\left|\lambda_{0}\right|=\gamma(T)$. If $T$ admits the following matrix representation

$$
T=\left[\begin{array}{ccc}
\lambda_{0} & \mu_{0} & C \\
0 & \lambda_{0} & B \\
0 & 0 & A
\end{array}\right]\{e, f\}^{\perp},
$$

where $e, f \in \mathscr{H}$ are two orthogonal normalized unit vectors, then given $\varepsilon>0$, there exists $K \in \mathscr{K}(\mathscr{H})$ with $\|K\|<\varepsilon$ such that $T+K \notin \mathcal{S}(\mathscr{N})$. 
Proof. It is easily seen that $\lambda_{0} \in \partial \sigma(T)$. Hence we can choose $\lambda_{1}, \mu_{1} \in \mathbb{C}$ such that $\left|\lambda_{1}\right|>\left|\lambda_{0}\right|, \mu_{1} \neq 0$ and $\left|\lambda_{1}-\lambda_{0}\right|+\left|\mu_{1}-\mu_{0}\right|<\varepsilon$. Then there exists $K \in \mathscr{K}(\mathscr{H})$ with $\|K\|<\varepsilon$ such that

$$
T+K=\left[\begin{array}{ccc}
\lambda_{1} & \mu_{1} & C \\
0 & \lambda_{1} & B \\
0 & 0 & A
\end{array}\right] \underset{\{e, f\}^{\perp}}{e} .
$$

Note that $\gamma(A) \leq \gamma(T)=\left|\lambda_{0}\right|<\left|\lambda_{1}\right|$, then $\lambda_{1} \notin \sigma(A)$ and $\gamma(T+K)=\left|\lambda_{1}\right|$. It remains to prove that $T+K \notin \mathcal{S}(\mathscr{N})$, that is, $T^{\prime} \notin \mathscr{N}$ for all $T^{\prime} \in \mathcal{S}(T+K)$.

Let $T^{\prime} \in \mathcal{S}(T+K)$ be fixed. Then $\sigma\left(T^{\prime}\right)=\sigma(T+K)$ and hence $\gamma\left(T^{\prime}\right)=$ $\gamma(T+K)=\left|\lambda_{1}\right|$. Since $T^{\prime} \sim T+K$, a similar argument as in the proof of Theorem 2.2 shows that, with respect to some suitably chosen $\operatorname{ONB}\{g, h\}$ of $\operatorname{ker}\left(T^{\prime}-\lambda_{1}\right)^{2}, T^{\prime}$ can be written as

$$
T^{\prime}=\left[\begin{array}{ccc}
\lambda_{1} & \mu_{2} & C_{1} \\
0 & \lambda_{1} & B_{1} \\
0 & 0 & A_{1}
\end{array}\right] \begin{gathered}
g \\
h \\
\{g, h\}^{\perp}
\end{gathered},
$$

where $\mu_{2} \neq 0$. One can easily shows that $\left\|T^{\prime}\right\|>\left|\lambda_{1}\right|=\gamma\left(T^{\prime}\right)$. Therefore $T^{\prime} \notin$ $\mathscr{N}$. Since $T^{\prime} \in \mathcal{S}(T+K)$ was arbitrarily chosen, we have $T+K \notin \mathcal{S}(\mathscr{N})$.

For convenience, we introduce a notation. We say that an operator $T \in$ $\mathscr{B}(\mathscr{H})$ has property (B), if each $\lambda \in \sigma(T)$ with $|\lambda|=\gamma(T)$ satisfies that $\lambda \in$ iso $\sigma(T)$ and $\operatorname{dim} \mathscr{H}(\lambda ; T)=1$.

Proposition 5.2. Let $T \in \mathscr{B}(\mathscr{H})$. If $T$ has property $(\mathrm{B})$, then $T$ is similar to a normaloid operator, that is, $T \in \mathcal{S}(\mathscr{N})$.

Proof. Since each $\lambda \in \sigma(T)$ with $|\lambda|=\gamma(T)$ is an isolated point of $\sigma(T)$, we deduce that $\Gamma=\{\lambda \in \sigma(T):|\lambda|=\gamma(T)\}$ consists of finite elements. It is obvious that $\Gamma$ is a nonempty clopen subset of $\sigma(T)$. Without loss of generality, we assume that $\left\{\lambda_{i}\right\}_{i=1}^{n}$ is an enumeration of points in $\Gamma$. Denote $\sigma=\sigma(T) \backslash \Gamma$. Then $\max \{|\lambda|: \lambda \in \sigma\}<\gamma(T)$.

By Corollary 3.7, $T$ admits the following matrix representation

$$
T=\left[\begin{array}{cc}
A & C \\
0 & B
\end{array}\right] \mathscr{\mathscr { H } ( \Gamma ; T ) ^ { \perp } ,},
$$

where $\sigma(A)=\Gamma=\left\{\lambda_{i}: 1 \leq i \leq n\right\}$ and $\sigma(B)=\sigma$. It is easily seen that $\gamma(B)=\max \{|\lambda|: \lambda \in \sigma\}$. Note that

$$
\mathscr{H}(\Gamma ; T)=\sum_{i=1}^{n} \mathscr{H}\left(\lambda_{i} ; T\right)
$$


and $\operatorname{dim} \mathscr{H}\left(\lambda_{i} ; T\right)=1$ for all $1 \leq i \leq n$, then $\operatorname{dim} \mathscr{H}(\Gamma ; T)=n$. With respect to some suitably chosen $\operatorname{ONB}\left\{e_{i}\right\}_{i=1}^{n}$ of $\mathscr{H}(\Gamma ; T), T$ can be written as

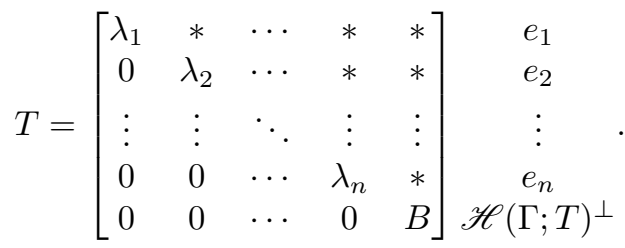

Note that $\left\{\lambda_{1}\right\},\left\{\lambda_{2}\right\}, \ldots,\left\{\lambda_{n}\right\}$ and $\sigma(B)$ are pairwise disjoint, then, by Lemma 3.6, we have

$$
T \sim\left[\begin{array}{ccccc}
\lambda_{1} & 0 & \cdots & 0 & 0 \\
0 & \lambda_{2} & \cdots & 0 & 0 \\
\vdots & \vdots & \ddots & \vdots & \vdots \\
0 & 0 & \cdots & \lambda_{n} & 0 \\
0 & 0 & \cdots & 0 & B
\end{array}\right] .
$$

Since $\gamma(B)=\max \{|\lambda|: \lambda \in \sigma\}<\gamma(T)=\left|\lambda_{1}\right|$, it follows from Lemma 3.2 that there exists an operator $B^{\prime}$ on $\mathscr{H}(\sigma ; T)$ such that $B^{\prime} \sim B$ and $\left\|B^{\prime}\right\|<\left|\lambda_{1}\right|$. Then

$$
T \sim\left[\begin{array}{ccccc}
\lambda_{1} & 0 & \cdots & 0 & 0 \\
0 & \lambda_{2} & \cdots & 0 & 0 \\
\vdots & \vdots & \ddots & \vdots & \vdots \\
0 & 0 & \cdots & \lambda_{n} & 0 \\
0 & 0 & \cdots & 0 & B^{\prime}
\end{array}\right] \triangleq T^{\prime}
$$

Note that $\left\|T^{\prime}\right\|=\left|\lambda_{1}\right|=\gamma\left(T^{\prime}\right)$, thus $T^{\prime} \in \mathscr{N}$ and we conclude that $T \in$ $\mathcal{S}(\mathscr{N})$.

Lemma 5.3 ([8], Corollary 1.6). Let $T \in \mathscr{B}(\mathscr{H})$. Assume that $\sigma$ is a clopen subset of $\sigma(T)$ and let $\Omega$ be a neighborhood of $\sigma$ such that $[\sigma(T) \backslash \sigma] \cap \bar{\Omega}=\emptyset$. Then there exists $\delta>0$ such that, for each $A \in \mathscr{B}(\mathscr{H})$ with $\|T-A\|<\delta$, $\sigma^{\prime} \triangleq \sigma(A) \cap \Omega \neq \emptyset$ and $\operatorname{dim} \mathscr{H}(\sigma ; T)=\operatorname{dim} \mathscr{H}\left(\sigma^{\prime} ; A\right)$.

Proposition 5.4. The set $\{X \in \mathscr{B}(\mathscr{H}): X$ has property $(\mathrm{B})\}$ is an open subset of $\mathscr{B}(\mathscr{H})$.

Proof. Let $T$ be an operator on $\mathscr{H}$ satisfying property (B). As seen in the proof of Proposition 5.2, we may directly assume that

(1) $\left\{\lambda_{i}: 1 \leq i \leq n\right\}$ is an enumeration of $\{\lambda \in \sigma(T):|\lambda|=\gamma(T)\}, \lambda_{i} \neq \lambda_{j}$ for $i \neq j, \lambda_{i} \in$ iso $\sigma(T)$ and $\operatorname{dim} \mathscr{H}\left(\lambda_{i} ; T\right)=1$ for all $i$, and

(2) $\sigma(T)=\left\{\lambda_{i}: 1 \leq i \leq n\right\} \cup \sigma, \sigma$ is a clopen subset of $\sigma(T), \max \{|\lambda|$ : $\lambda \in \sigma\}<\gamma(T)=\left|\lambda_{1}\right|$.

Arbitrarily choose a positive number $r$ with $\max \{|\lambda|: \lambda \in \sigma\}<r<\gamma(T)$. Obviously, there exists $\varepsilon>0$ satisfying that $\overline{B_{r}(0)}, \overline{B_{\varepsilon}\left(\lambda_{1}\right)}, \overline{B_{\varepsilon}\left(\lambda_{2}\right)}, \ldots$ and $\overline{B_{\varepsilon}\left(\lambda_{n}\right)}$ are pairwise disjoint. 
Denote $\Omega_{i}=B_{\varepsilon}\left(\lambda_{i}\right)(1 \leq i \leq n), \Omega_{0}=B_{r}(0)$ and $\sigma_{i}=\sigma(T) \cap \Omega_{i}(0 \leq i \leq n)$. By the upper semi-continuity of spectrum and Lemma 5.3, there exists $\delta>0$ such that, for each $A \in \mathscr{B}(\mathscr{H})$ with $\|T-A\|<\delta$, the following conditions hold:

(3) $\sigma(A) \subset \cup_{i=0}^{n} \Omega_{i}$;

(4) $\sigma_{i}^{\prime}=\sigma(A) \cap \Omega_{i} \neq \emptyset$ and $\operatorname{dim} \mathscr{H}\left(\sigma_{i}^{\prime} ; A\right)=\operatorname{dim} \mathscr{H}\left(\sigma_{i} ; T\right)$ for all $0 \leq i \leq n$.

Let $A$ be fixed. To complete the proof, we need only prove that $A$ has property (B). Obviously, $\sigma(A)=\cup_{i=0}^{n} \sigma_{i}^{\prime}, \sigma_{0}^{\prime} \subset B_{r}(0)$ and $\min \left\{|\lambda|: \lambda \in \sigma_{i}^{\prime}\right\}>r$ for all $1 \leq i \leq n$. For each $i, 1 \leq i \leq n$, since $\operatorname{dim} \mathscr{H}\left(\sigma_{i} ; T\right)=1(1 \leq i \leq n)$, we obtain $\operatorname{dim} \mathscr{H}\left(\sigma_{i}^{\prime} ; A\right)=1$ and $\sigma_{i}^{\prime}$ is a singleton. It follows that $\{\lambda \in \sigma(A):|\lambda|=$ $\gamma(A)\} \subseteq \cup_{i=1}^{n} \sigma_{i}^{\prime}$ and hence $A$ has property (B). This completes the proof.

We say that an operator $T \in \mathscr{B}(\mathscr{H})$ has property $(\mathrm{C})$, if there exists a finite subset $\Gamma$ of $\sigma_{1}(T)$ such that $[\sigma(T) \backslash \Gamma] \subset[\operatorname{conv} \Gamma]^{\circ}$. Then, using a similar argument as in Proposition 5.4, one can prove the following result.

Corollary 5.5. The set $\{X \in \mathscr{B}(\mathscr{H}): X$ has property $(\mathrm{C})\}$ is an open subset of $\mathscr{B}(\mathscr{H})$.

Now we are in a position to prove Theorem 2.4.

Proof of Theorem 2.4. The proof for the sufficiency follows immediately from Propositions 5.2, 5.4. We need only prove the necessity.

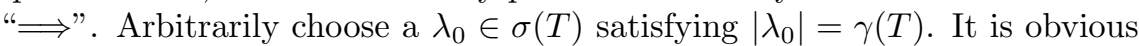
that $\lambda_{0} \in \partial \sigma(T)$. For a proof by contrary, we assume that either $\lambda_{0} \notin$ iso $\sigma(T)$, or $\lambda_{0} \in$ iso $\sigma(T)$ and $\operatorname{dim} \mathscr{H}\left(\lambda_{0} ; T\right)>1$.

Case 1. $\lambda_{0} \notin$ iso $\sigma(T)$. In this case, it follows from Lemma 4.5 that $\lambda_{0} \in \sigma_{\text {lre }}(T)$. Then, given $\varepsilon>0$, it follows from Lemma 4.4 that there exists $K_{1} \in \mathscr{K}(\mathscr{H})$ with $\left\|K_{1}\right\|<\varepsilon / 2$ such that

$$
T+K_{1}=\left[\begin{array}{ccc}
\lambda_{0} & \mu_{0} & C \\
0 & \lambda_{0} & B \\
0 & 0 & A
\end{array}\right] \begin{gathered}
e \\
f e, f\}^{\perp}
\end{gathered},
$$

where $\sigma(A)=\sigma(T)$. It is obvious that $\gamma\left(T+K_{1}\right)=\left|\lambda_{0}\right|$. According to Proposition 5.1, there exists $K_{2} \in \mathscr{K}(\mathscr{H})$ with $\left\|K_{2}\right\|<\varepsilon / 2$ such that $T+$ $K_{1}+K_{2} \notin \mathcal{S}(\mathscr{N})$. Thus we have proved that for arbitrarily given $\varepsilon>0$ there exists $K \in \mathscr{K}(\mathscr{H})$ with $\|K\|<\varepsilon$ such that $T+K \notin \mathcal{S}(\mathscr{N})$. Therefore $T \notin \mathcal{S}(\mathscr{N})^{\circ}$, a contradiction.

Case 2. $\lambda_{0} \in$ iso $\sigma(T)$ and $\operatorname{dim} \mathscr{H}\left(\lambda_{0} ; T\right)>1$. In this case, we may directly assume that $\operatorname{dim} \mathscr{H}\left(\lambda_{0} ; T\right)<\infty$. In fact, if $\operatorname{dim} \mathscr{H}\left(\lambda_{0} ; T\right)=\infty$, then $\lambda_{0} \in \sigma_{\text {lre }}(T)$. Using a same argument as in Case 1 , we can prove that $T \notin \mathcal{S}(\mathscr{N})^{\circ}$, a contradiction.

Now we assume that $n=\operatorname{dim} \mathscr{H}\left(\lambda_{0} ; T\right)$ and $1<n<\infty$. Since $\lambda_{0} \in$ iso $\sigma(T)$, it follows from Corollary 3.7 that $T$ can be written as

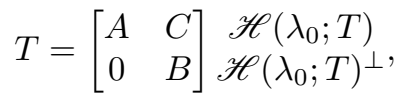


where $\sigma(A)=\left\{\lambda_{0}\right\}$ and $\sigma(B)=\sigma(T) \backslash\left\{\lambda_{0}\right\}$. Note that $1<\operatorname{dim} \mathscr{H}\left(\lambda_{0} ; T\right)<\infty$, then, with respect to some ONB $\left\{e_{1}, e_{2}, \ldots, e_{n}\right\}$ of $\mathscr{H}\left(\lambda_{0} ; T\right), T$ can be written as

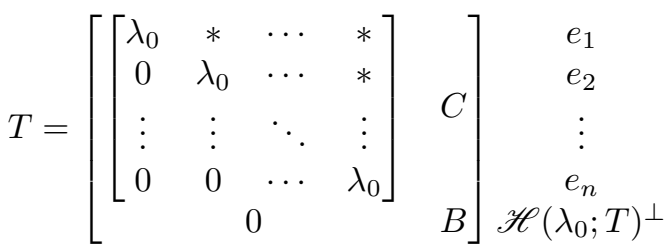

$$
\begin{aligned}
& \triangleq\left[\begin{array}{ccc}
\lambda_{0} & \mu_{0} & * \\
0 & \lambda_{0} & * \\
0 & 0 & *
\end{array}\right] \begin{array}{c}
e_{1} \\
e_{2} \\
\left\{e_{1}, e_{2}\right\}^{\perp}
\end{array} .
\end{aligned}
$$

Then, according to Proposition 5.1, we obtain $T \notin \mathcal{S}(\mathscr{N})^{\circ}$, a contradiction.

Corollary 5.6. Let $T \in \mathscr{B}(\mathscr{H})$. If $T \notin \mathcal{S}(\mathscr{N})^{\circ}$, then, given $\varepsilon>0$, there exists $K \in \mathscr{K}(\mathscr{H})$ with $\|K\|<\varepsilon$ such that $T+K \notin \mathcal{S}(\mathscr{N})$.

\section{Proofs of Theorems 2.5, 2.6}

This section is mainly devoted to the proofs of Theorems 2.5, 2.6. As an application, we construct an operator to give a negative answer to a problem posed by W. Y. Lee [10].

Proposition 6.1. Let $T \in \mathscr{B}(\mathscr{H})$. Then the following are equivalent:

(i) $\left[\sigma(T) \backslash \sigma_{1}(T)\right] \subset\left[\operatorname{conv} \sigma_{1}(T)\right]^{\circ}$;

(ii) there exists a finite subset $\Gamma$ of $\sigma_{1}(T)$ such that $[\sigma(T) \backslash \Gamma] \subset[\operatorname{conv} \Gamma]^{\circ}$.

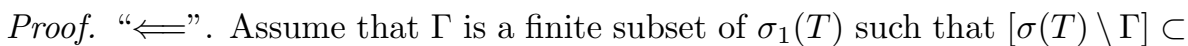
$[\text { conv } \Gamma]^{\circ}$. Then $\left[\sigma(T) \backslash \sigma_{1}(T)\right] \subset[\sigma(T) \backslash \Gamma]$, conv $\Gamma \subset \operatorname{conv} \sigma_{1}(T)$ and hence $\left[\sigma(T) \backslash \sigma_{1}(T)\right] \subset\left[\operatorname{conv} \sigma_{1}(T)\right]^{\circ}$.

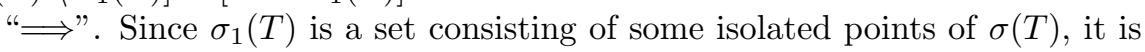
easy to see that $\sigma(T) \backslash \sigma_{1}(T)$ is compact and card $\sigma_{1}(T) \leq \aleph_{0}$. Without loss of generality, we assume that $\left\{\lambda_{i}\right\}_{i=1}^{\infty}$ is an enumeration of $\sigma_{1}(T)$.

For each $n \in \mathbb{N}$, denote $\Gamma_{n}=\left\{\lambda_{i}: 1 \leq i \leq n\right\}$. Then conv $\Gamma_{n} \subset \operatorname{conv} \Gamma_{n+1}$ and conv $\sigma_{1}(T)=\cup_{i=1}^{\infty}\left[\operatorname{conv} \Gamma_{i}\right]$. Note that each conv $\Gamma_{n}$ is a closed convex set, then one can prove that $\left[\operatorname{conv} \sigma_{1}(T)\right]^{\circ}=\cup_{i=1}^{\infty}\left[\operatorname{conv} \Gamma_{i}\right]^{\circ}$.

Since $\left[\sigma(T) \backslash \sigma_{1}(T)\right] \subset\left[\operatorname{conv} \sigma_{1}(T)\right]^{\circ}$, it follows that $\left\{\left[\operatorname{conv} \Gamma_{i}\right]^{\circ}: i \geq 1\right\}$ is an open cover of the compact set $\sigma(T) \backslash \sigma_{1}(T)$. Then there exists some $n_{0}$ such that $\left[\sigma(T) \backslash \sigma_{1}(T)\right] \subset\left[\operatorname{conv} \Gamma_{n_{0}}\right]^{\circ}$.

On the other hand, it is easy to see that each limit point of $\sigma_{1}(T)$ lies in $\sigma(T) \backslash \sigma_{1}(T)$. Hence $\Gamma \triangleq\left\{\lambda \in \sigma_{1}(T): \lambda \notin\left[\text { conv } \Gamma_{n_{0}}\right]^{\circ}\right\}$ consists of at most finite points. Note that $\Gamma_{n_{0}} \subset \Gamma$, then one can deduce that $[\sigma(T) \backslash \Gamma] \subset[\operatorname{conv} \Gamma]^{\circ}$.

Lemma 6.2. Let $T \in \mathscr{B}(\mathscr{H})$. If $\sigma(T) \backslash \sigma_{1}(T)$ is not contained in [conv $\left.\sigma_{1}(T)\right]^{\circ}$, then, given $\varepsilon>0$, there exists $K \in \mathscr{K}(\mathscr{H})$ with $\|K\|<\varepsilon$ such that $\sigma(S) \backslash \sigma_{1}(S)$ is not contained in $\overline{\text { conv } \sigma_{1}(S)}$, where $S=T+K$. 
Proof. Obviously, we may directly assume that $\left[\sigma(T) \backslash \sigma_{1}(T)\right] \subset \overline{\operatorname{conv} \sigma_{1}(T)}$ (otherwise $K=0$ satisfies all requirements). Hence we can choose a $\lambda_{0} \in$ $\left[\sigma(T) \backslash \sigma_{1}(T)\right] \cap\left[\partial\left(\operatorname{conv} \sigma_{1}(T)\right)\right]$. It is easy to see that $\lambda_{0} \in \partial \sigma(T)$. Then either $\lambda_{0} \in \sigma_{0}(T)$ and $\operatorname{dim} \mathscr{H}\left(\lambda_{0} ; T\right)>1$, or $\lambda_{0} \in \sigma_{\text {lre }}(T)$. Also, for given $\varepsilon>0$, we can choose $\lambda_{1} \in \mathbb{C}$ with $\left|\lambda_{0}-\lambda_{1}\right|<\varepsilon / 2$ such that $\lambda_{1} \notin \overline{\operatorname{conv} \sigma_{1}(T)}$.

Case 1. $\lambda_{0} \in \sigma_{0}(T)$ and $\operatorname{dim} \mathscr{H}\left(\lambda_{0} ; T\right)>1$. In this case, it follows from Corollary 3.7 that

$$
T=\left[\begin{array}{cc}
A & C \\
0 & B
\end{array}\right] \begin{aligned}
& \mathscr{H}\left(\lambda_{0} ; T\right) \\
& \mathscr{H}\left(\lambda_{0} ; T\right)^{\perp}
\end{aligned}
$$

where $\sigma(A)=\left\{\lambda_{0}\right\}$ and $\sigma(B)=\sigma(T) \backslash\left\{\lambda_{0}\right\}$. Since $\operatorname{dim} \mathscr{H}\left(\lambda_{0} ; T\right)<\infty$, it is obvious that there exists $K \in \mathscr{K}(\mathscr{H})$ with $\|K\|<\varepsilon$ such that

$$
T+K=\left[\begin{array}{cc}
\bar{A} & C \\
0 & B
\end{array}\right] \stackrel{\mathscr{H}\left(\lambda_{0} ; T\right)}{\mathscr{H}\left(\lambda_{0} ; T\right)^{\perp}},
$$

where $\sigma(\bar{A})=\left\{\lambda_{1}\right\}$. Denote $S \triangleq T+K$. It is easy to verify that $\sigma(S)=$ $\sigma(B) \cup\left\{\lambda_{1}\right\}, \sigma_{1}(S)=\sigma_{1}(B)=\sigma_{1}(T)$. Then $\lambda_{1} \notin \overline{\operatorname{conv} \sigma_{1}(S)}$ and $K$ satisfies all requirements.

Case 2. $\lambda_{0} \in \sigma_{\text {lre }}(T)$. By Lemma 4.4, for arbitrarily given $\varepsilon>0$, there exists $K_{1} \in \mathscr{K}(\mathscr{H})$ with $\left\|K_{1}\right\|<\varepsilon / 2$ such that

$$
T+K_{1}=\left[\begin{array}{ccc}
\lambda_{0} & \mu_{0} & D \\
0 & \lambda_{0} & C \\
0 & 0 & B
\end{array}\right] \begin{gathered}
e \\
f \\
\{e, f\}^{\perp}
\end{gathered},
$$

where $e, f$ are orthogonal unit vectors in $\mathscr{H}, \sigma(B)=\sigma(T)$ and $\sigma_{1}(B)=\sigma_{1}(T)$. Since $\left|\lambda_{0}-\lambda_{1}\right|<\varepsilon / 2$, there exists $K \in \mathscr{K}(\mathscr{H})$ with $\|K\|<\varepsilon$ such that

$$
T+K=\left[\begin{array}{ccc}
\lambda_{1} & \mu_{0} & D \\
0 & \lambda_{1} & C \\
0 & 0 & B
\end{array}\right] \begin{gathered}
e \\
f e, f\}^{\perp}
\end{gathered} .
$$

Denote $S=T+K$. Then, as in Case 1 , one can easily verify that $K$ satisfies all requirements.

If $\sigma$ is a nonempty compact subset of $\mathbb{C}$ and $\mu \in \mathbb{C}$, then we denote $\Upsilon(\mu, \sigma)=$ $\min \{r \geq 0:|\lambda-\mu| \leq r$ for all $\lambda \in \sigma\}$. The following lemma is a direct observation.

Lemma 6.3. Let $\sigma$ be a nonempty compact subset of $\mathbb{C}$. Assume that $\Gamma \subset \sigma$

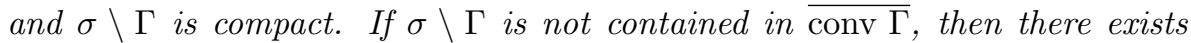
$\mu \in \mathbb{C}$ such that $\{\lambda \in \sigma:|\mu-\lambda|=\Upsilon(\mu, \sigma)\} \subset \sigma \backslash \Gamma$.

A nonempty bounded open subset $\Omega$ of $\mathbb{C}$ is called a Cauchy domain, if $\Omega$ has finitely many components and $\partial \Omega$ consists of finitely many, piecewise disjoint, rectifiable Jordan curves. If, in addition, $\Omega$ is connected, we say that $\Omega$ is a Cauchy region. 
Proof of Theorem 2.5. By Proposition 6.1, "(ii) $\Longleftrightarrow($ iii)" is obvious. Hence it suffices to prove that "(i) $\Longrightarrow$ (ii)" and "(iii) $\Longrightarrow($ i $)$ ".

"(i) $\Longrightarrow\left(\right.$ ii)". For a proof by contrary, we assume that $\sigma(T) \backslash \sigma_{1}(T)$ is not contained in $\left[\operatorname{conv} \sigma_{1}(T)\right]^{\circ}$. Then, according to Lemma 6.2, we may directly assume that $\sigma(T) \backslash \sigma_{1}(T)$ is not contained in $\overline{\text { conv } \sigma_{1}(T)}$. By Lemma 6.3, there exists $\mu_{0} \in \mathbb{C}$ and $\lambda_{0} \in\left[\sigma(T) \backslash \sigma_{1}(T)\right]$ such that $\left|\mu_{0}-\lambda_{0}\right|=\Upsilon\left(\mu_{0}, \sigma(T)\right)$. Note that

$$
\Upsilon\left(\mu_{0}, \sigma(T)\right)=\max \left\{\left|\mu_{0}-\lambda\right|: \lambda \in \sigma(T)\right\}=\gamma\left(\mu_{0}-T\right),
$$

then we have shown that there exists $\mu_{0}-\lambda_{0} \in\left[\sigma\left(\mu_{0}-T\right) \backslash \sigma_{1}\left(\mu_{0}-T\right)\right]$ with $\left|\mu_{0}-\lambda_{0}\right|=\gamma\left(\mu_{0}-T\right)$. By Theorem 2.4 and Corollary 5.6, $\mu_{0}-T \notin \mathcal{S}(\mathscr{N})^{\circ}$ and, for arbitrarily given $\varepsilon>0$, there exists $K \in \mathscr{K}(\mathscr{H})$ with $\|K\|<\varepsilon$ such that $T-\mu_{0}+K \notin \mathcal{S}(\mathscr{N})$. Hence $T+K \notin \mathcal{S}(\mathscr{T})$. Since $\varepsilon>0$ is arbitrary, we conclude that $T \notin \mathcal{S}(\mathscr{T})^{\circ}$, a contradiction.

"(iii) $\Longrightarrow($ i)". By Corollary 5.5, $\{X \in \mathscr{B}(\mathscr{H}): X$ satisfies (iii) $\}$ is an open subset of $\mathscr{B}(\mathscr{H})$. Then it suffices to prove that if $T$ satisfies (iii), then $T \in$ $\mathcal{S}(\mathscr{T})$.

Assume that $\Gamma$ is a finite subset of $\sigma_{1}(T)$ satisfying $[\sigma(T) \backslash \Gamma] \subset[\operatorname{conv} \Gamma]^{\circ}$. We may assume that $\Gamma=\left\{\lambda_{i}: 1 \leq i \leq n\right\}$, where $\lambda_{i} \neq \lambda_{j}$ for $i \neq j$. By Corollary 3.7, $T$ can be written as

$$
T=\left[\begin{array}{ccccc}
\lambda_{1} & * & * & \cdots & * \\
& \lambda_{2} & * & \cdots & * \\
& & \ddots & \ddots & \vdots \\
& & & \lambda_{n} & * \\
& & & & A
\end{array}\right] \begin{gathered}
e_{1} \\
e_{2} \\
\vdots \\
e_{n} \\
\left.e_{i}\right\}^{\perp}
\end{gathered}
$$

where $\left\{e_{i}\right\}$ are orthogonal normalized vectors and $\sigma(A) \subset[\operatorname{conv} \Gamma]^{\circ}$.

We can choose a Cauchy region $\Phi$ such that $\sigma(A) \subset \Phi \subset \bar{\Phi} \subset[\operatorname{conv} \Gamma]^{\circ}$. By Theorem 1.2, there exists $A^{\prime}$ similar to $A$ such that $\bar{\Phi}$ is a spectral set of $A^{\prime}$. Then $\left\|\lambda-A^{\prime}\right\|=\max \{|\lambda-\mu|: \mu \in \bar{\Phi}\}$ for all $\lambda \in \mathbb{C}$. Since $\left\{\lambda_{1}\right\}, \ldots,\left\{\lambda_{n}\right\}, \sigma(A)$ are pairwise disjoint, it follows from Lemma 3.6 that $T \sim\left[\oplus_{i=1}^{n} \lambda_{i}\right] \oplus A^{\prime} \triangleq T^{\prime}$. Now it remains to prove that $T^{\prime} \in \mathscr{T}$, that is, $\left\|\lambda-T^{\prime}\right\|=\gamma\left(\lambda-T^{\prime}\right)$ for all $\lambda \in \mathbb{C}$.

Let $\lambda \in \mathbb{C}$ be fixed. Since $\sigma\left(T^{\prime}\right)=\sigma\left(A^{\prime}\right) \cup \Gamma$ and $\bar{\Phi} \subset[\text { conv } \Gamma]^{\circ}$, one can easily infer that

$$
\begin{aligned}
\left\|\lambda-A^{\prime}\right\| & =\max \{|\lambda-\mu|: \mu \in \bar{\Phi}\} \\
& <\max \left\{\left|\lambda-\lambda_{i}\right|: 1 \leq i \leq n\right\} \\
& =\left\|\lambda-\oplus_{i=1}^{n} \lambda_{i}\right\| .
\end{aligned}
$$

Hence

$$
\begin{aligned}
\left\|\lambda-T^{\prime}\right\| & =\max \left\{\left\|\lambda-\oplus_{i=1}^{n} \lambda_{i}\right\|,\left\|\lambda-A^{\prime}\right\|\right\} \\
& =\left\|\lambda-\oplus_{i=1}^{n} \lambda_{i}\right\|=\max \left\{\left|\lambda-\lambda_{i}\right|: 1 \leq i \leq n\right\} \\
& \leq \max \left\{|\lambda-\mu|: \mu \in \sigma\left(T^{\prime}\right)\right\}=\gamma\left(\lambda-T^{\prime}\right)
\end{aligned}
$$


This implies that $T^{\prime} \in \mathscr{T}$. Thus we complete the proof.

Corollary 6.4. Let $T \in \mathscr{B}(\mathscr{H})$. If $T \notin \mathcal{S}(\mathscr{T})^{\circ}$, then, given $\varepsilon>0$, there exists $K \in \mathscr{K}(\mathscr{H})$ with $\|K\|<\varepsilon$ such that $T+K \notin \mathcal{S}(\mathscr{T})$.

Proposition 6.5. Let $T \in \mathscr{B}(\mathscr{H})$ and $\Gamma=\left\{\lambda_{i}: 1 \leq i \leq n\right\}$ be a finite subset of $\mathbb{C}$. If conv $\Gamma$ contains each $\lambda \in \mathbb{C}$ with $|\lambda| \leq\|T\|$, then $S=\left[\oplus_{i=1}^{n} \lambda_{i}\right] \oplus T$ is a transloid operator on $\mathbb{C}^{n} \oplus \mathscr{H}$.

Proof. Arbitrarily choose a $\mu \in \mathbb{C}$. It suffices to prove that $\|\mu-S\|=\gamma(\mu-S)$. Since $\{\lambda \in \mathbb{C}:|\lambda| \leq\|T\|\} \subset[\operatorname{conv} \Gamma]$, one can easily infer that

$$
\begin{aligned}
\|\mu-T\| & \leq|\mu|+\|T\| \leq \max \left\{\left|\mu-\lambda_{i}\right|: 1 \leq i \leq n\right\} \\
& \leq\left\|\mu-\oplus_{i=1}^{n} \lambda_{i}\right\| .
\end{aligned}
$$

Hence

$$
\begin{aligned}
\|\mu-S\| & =\max \left\{\left\|\mu-\oplus_{i=1}^{n} \lambda_{i}\right\|,\|\mu-T\|\right\} \\
& =\left\|\mu-\oplus_{i=1}^{n} \lambda_{i}\right\|=\max \left\{\left|\mu-\lambda_{i}\right|: 1 \leq i \leq n\right\} \\
& \leq \max \{|\mu-\lambda|: \lambda \in \sigma(S)\}=\gamma(\mu-S) .
\end{aligned}
$$

Thus we obtain $\|\mu-S\|=\gamma(\mu-S)$.

Proof of Theorem 2.6. Since $\mathscr{T} \subset \mathscr{N}$, we need only prove that $\mathscr{B}(\mathscr{H}) \subset \mathscr{T}+$ $\mathscr{K}(\mathscr{H})$. Let $T \in \mathscr{B}(\mathscr{H})$ be fixed. It suffices to prove $T \in \mathscr{T}+\mathscr{K}(\mathscr{H})$. Arbitrarily choose an $\mathrm{ONB}\left\{e_{i}\right\}_{i=1}^{\infty}$ of $\mathscr{H}$. Assume that $T$ admits the following matrix representation

$$
T=\left[\begin{array}{cccc}
t_{11} & t_{12} & t_{13} & \cdots \\
t_{21} & t_{22} & t_{23} & \cdots \\
t_{31} & t_{32} & t_{33} & \cdots \\
\vdots & \vdots & \vdots & \ddots
\end{array}\right] \begin{aligned}
& e_{1} \\
& e_{2} \\
& e_{3} \\
& \vdots
\end{aligned} \triangleq\left[\begin{array}{cc}
B & C \\
D & A
\end{array}\right] M_{M^{\perp}}^{M}
$$

where $M$ is the subspace of $\mathscr{H}$ spanned by $e_{1}, e_{2}, e_{3}$. It is easy to see that $B, C, D$ are compact. Then there exists $K_{1} \in \mathscr{K}(\mathscr{H})$ such that

$$
T+K_{1}=\left[\begin{array}{ll}
0 & 0 \\
0 & A
\end{array}\right] \begin{gathered}
M \\
M^{\perp}
\end{gathered}
$$

Obviously we can find $\lambda_{1}, \lambda_{2}, \lambda_{3} \in \mathbb{C}$ such that conv $\left\{\lambda_{i}: 1 \leq i \leq 3\right\}$ contains each $\lambda \in \mathbb{C}$ with $|\lambda| \leq\|A\|$. Set

$$
K_{2}=\left[\begin{array}{llll}
\lambda_{1} & & & \\
& \lambda_{2} & & \\
& & \lambda_{3} & \\
& & & 0
\end{array}\right] \begin{gathered}
e_{1} \\
e_{2} \\
e_{3} \\
M^{\perp}
\end{gathered}, K=K_{1}+K_{2} .
$$


Then $K \in \mathscr{K}(\mathscr{H})$ and

$$
T+K=\left[\begin{array}{llll}
\lambda_{1} & & & \\
& \lambda_{2} & & \\
& & \lambda_{3} & \\
& & & A
\end{array}\right] \begin{gathered}
e_{1} \\
e_{2} \\
e_{3} \\
M^{\perp}
\end{gathered} .
$$

By Proposition 6.5, we deduce that $T+K \in \mathscr{T}$. Hence $T \in \mathscr{T}+\mathscr{K}(\mathscr{H})$.

We shall conclude this section with an application of Theorem 2.5 to a problem posed by W. Y. Lee, concerning Weyl's theorem for transloid operators. Following Coburn, we say that Weyl's theorem holds for an operator $T \in \mathscr{B}(\mathscr{H})$ if $\sigma(T) \backslash \sigma_{w}(T)=\pi_{00}(T)$, where $\pi_{00}(T)=\{\lambda \in$ iso $\sigma(T): 0<$ nul $(\lambda-T)<\infty\}$ and $\sigma_{w}(T)=\sigma_{\text {lre }}(T) \cup\left\{\lambda \in \rho_{s-F}(T)\right.$ : ind $\left.(\lambda-T) \neq 0\right\}$ is the Weyl spectrum of $T$. $T$ is said to have the single-valued extension property (SVEP for short), denoted by $T \in$ SVEP, if, for every open set $U \subseteq \mathbb{C}$, the only analytic solution $f(\cdot): U \rightarrow \mathscr{H}$ of the equation $(T-\lambda) f(\lambda)=0$ for all $\lambda \in U$ is the zero function on $U$. W. Y. Lee posed the following problem in [10].

Problem 6.6 (Problem 2.1, [10]). If $T \in \mathscr{B}(\mathscr{H})$ is transaloid and has the SVEP, does Weyl's theorem hold for $T$ ?

Since Weyl's theorem holds for reguloid operators with SVEP, W. Y. Lee attempted to give a positive answer to Problem 6.6 by proving that "transloid $\Rightarrow$ reguloid". We construct the following example to give a negative answer to Problem 6.6.

Example 6.7. Suppose that $\left\{e_{i}\right\}_{i=1}^{\infty}$ is an ONB of $\mathscr{H}$ and denote

$$
K=\left[\begin{array}{ccccc}
0 & 1 / 4 & & & \\
& 0 & 1 / 5 & & \\
& & 0 & 1 / 6 & \\
& & & 0 & \ddots \\
& & & & \ddots
\end{array}\right] \begin{aligned}
& e_{1} \\
& e_{2} \\
& e_{3} \\
& e_{4} \\
& \vdots \\
&
\end{aligned}
$$

By Proposition 6.5, $T$ is transloid. On the other hand, it is obvious that $T \in \operatorname{SveP}, \sigma(T)=\{0,1, i,-1-i\}=\pi_{00}(T)$ and $\sigma_{w}(T)=\{0\}$. Hence Weyl's theorem does not hold for $T$. This also means that "transloid" does not imply "reguloid".

\section{Proof of Theorem 2.7}

Given a subset $\sigma$ of $\mathbb{C}$ and $\varepsilon>0$, denote $\sigma_{\varepsilon}=\{\lambda \in \mathbb{C}: \operatorname{dist}(\lambda, \sigma)<\varepsilon\}$, where $\operatorname{dist}(\lambda, \sigma)$ is the distance between $\lambda$ and $\sigma$.

Proposition 7.1. Let $T \in \mathscr{B}(\mathscr{H})$ satisfy $\sigma(T)=\sigma_{w}(T)$. Suppose that $\Omega$ is a Cauchy region such that $\sigma(T) \subset \Omega \subset \bar{\Omega} \subset \sigma(T)_{\varepsilon}$, where $\varepsilon>0$. Then there exists $K \in \mathscr{K}(\mathscr{H})$ with $\|K\|<2 \varepsilon$ such that $T+K \in \mathcal{S}(\mathscr{T})^{\circ}$. 
Proof. Assume that $\Gamma$ is the boundary of the unbounded component of $\mathbb{C} \backslash \bar{\Omega}$. It is obvious that $\Gamma$ is a closed Jordan curve and $\Gamma \subset \partial \Omega$. Denote by $\Omega_{0}$ the bounded simply-connected region surrounding by $\Gamma$. Then $\Gamma=\partial \Omega_{0}, \Gamma \subset \sigma(T)_{\varepsilon}$ and $\Omega \subset \Omega_{0}$.

Since $\sigma(T) \subset \Omega_{0}$, obviously we can choose finite pairwise distinct points $\lambda_{1}, \lambda_{2}, \ldots, \lambda_{n}$ in $\Gamma$ such that $\sigma(T) \subset\left[\operatorname{conv}\left\{\lambda_{i}: 1 \leq i \leq n\right\}\right]^{\circ}$. For each $1 \leq i \leq n$, note that $\operatorname{dist}\left(\lambda_{i}, \sigma(T)\right)<\varepsilon$, then there exists $\mu_{i} \in \partial \sigma(T)$ such that $\left|\lambda_{i}-\mu_{i}\right|<\varepsilon . \quad \sigma(T)=\sigma_{w}(T)$ implies that $\partial \sigma(T) \subset \sigma_{\text {lre }}(T)$ and hence $\mu_{i} \in \sigma_{\text {lre }}(T)$ for all $i$. By Lemma 4.4, there exists $K_{1} \in \mathscr{K}(\mathscr{H})$ with $\left\|K_{1}\right\|<\varepsilon$ such that

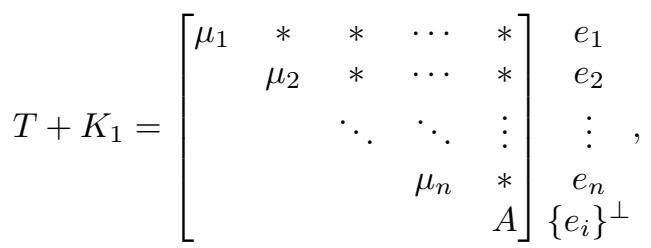

where $\left\{e_{i}\right\}$ are orthogonal normalized vectors and $\sigma(A)=\sigma(T)$. Obviously, there exists $K_{2} \in \mathscr{K}(\mathscr{H})$ with $\left\|K_{2}\right\|<\varepsilon$ such that

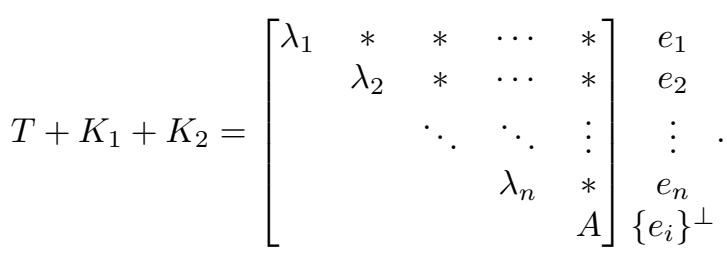

Denote $K=K_{1}+K_{2}$ and $\sigma_{1}=\left\{\lambda_{i}: 1 \leq i \leq n\right\}$. It is easy to see that $\sigma_{1} \subset \sigma_{1}(T+K)$ and $\left[\sigma(T+K) \backslash \sigma_{1}\right]=\sigma(A) \subset\left[\operatorname{conv} \sigma_{1}\right]^{\circ}$. According to Theorem 2.5, we obtain $T+K \in \mathcal{S}(\mathscr{T})^{\circ}$ and $K$ satisfies all requirements.

Theorem 7.2. Let $T \in \mathscr{B}(\mathscr{H})$ satisfy $\sigma(T)=\sigma_{w}(T)$. Then, given $\varepsilon>0$, there exists $K \in \mathscr{K}(\mathscr{H})$ with $\|K\|<\varepsilon$ such that $T+K \in \mathcal{S}(\mathscr{T})^{\circ}$.

Proof. For given $\varepsilon>0$, we can choose a Cauchy domain $\Omega$ such that $\sigma(T) \subset$ $\Omega \subset \bar{\Omega} \subset \sigma(T)_{\varepsilon / 4}$. Assume that $\Omega_{1}, \ldots, \Omega_{n}$ is an enumeration of the components of $\Omega$. For each $i$, set $\sigma_{i}=\sigma(T) \cap \Omega_{i}$. Then $\sigma_{i}(1 \leq i \leq n)$ are pairwise disjoint clopen subsets of $\sigma(T)$ and $\sigma(T)=\cup_{i=1}^{n} \sigma_{i}$. We may assume that $\sigma_{i} \neq \emptyset$ for all $i$.

By Corollary 4.7, $T$ admits the following matrix representation

$$
T=\left[\begin{array}{cccc}
T_{1} & * & \cdots & * \\
& T_{2} & \cdots & * \\
& & \ddots & \vdots \\
& & & T_{n}
\end{array}\right] \begin{gathered}
M_{1} \\
M_{2} \\
\vdots \\
M_{n}
\end{gathered},
$$

where $\oplus_{i=1}^{n} M_{i}=\mathscr{H}$ and $\sigma\left(T_{i}\right)=\sigma_{i}(1 \leq i \leq n)$. Also it can be required that $\sigma_{w}\left(T_{i}\right)=\sigma\left(T_{i}\right)=\sigma_{i} \subset \Omega_{i} \subset \sigma\left(T_{i}\right)_{\varepsilon / 4}(1 \leq i \leq n)$. 
Let $i(1 \leq i \leq n)$ be fixed. Note that $\Omega_{i}$ is a Cauchy region, then, by Proposition 7.1, there exists $K_{i}$ compact with $\left\|K_{i}\right\|<\varepsilon / 2$ and a finite subset $\Gamma_{i}$ of $\sigma_{1}\left(T_{i}+K_{i}\right)$ such that $\left[\sigma\left(T_{i}+K_{i}\right) \backslash \Gamma_{i}\right] \subset\left[\operatorname{conv} \Gamma_{i}\right]^{\circ}$. Also $\Gamma_{i}$ can be required to satisfy $\Gamma_{i} \subset \Omega_{i}$.

For each $i$, set $A_{i}=T_{i}+K_{i}$ and

$$
K=\left[\begin{array}{llll}
K_{1} & & & \\
& K_{2} & & \\
& & \ddots & \\
& & & K_{n}
\end{array}\right] \begin{gathered}
M_{1} \\
M_{2} \\
\vdots \\
M_{n}
\end{gathered} .
$$

Then $K \in \mathscr{K}(\mathscr{H}),\|K\|<\varepsilon$ and

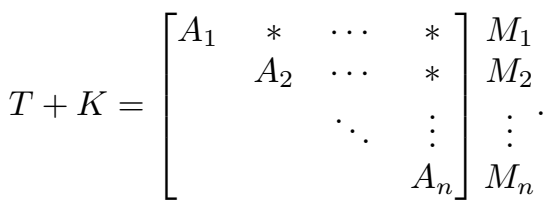

Denote $\Gamma=\cup_{i=1}^{n} \Gamma_{i}$. Note that $\Gamma$ is a finite subset of $\sigma_{1}(T+K)$ and $[\sigma(T+K) \backslash$ $\Gamma] \subset[\operatorname{conv} \Gamma]^{\circ}$, then it follows from Theorem 2.5 that $T+K \in \mathcal{S}(\mathscr{T})^{\circ}$.

Lemma 7.3 ([8], Theorem 3.48). Let $T \in \mathscr{B}(\mathscr{H})$. Then, given $\varepsilon>0$, there exists $K \in \mathscr{K}(\mathscr{H})$ such that

$$
\|K\|<\varepsilon+\max \left\{\operatorname{dist}\left[\lambda, \partial \rho_{s-F}(T)\right]: \lambda \in \sigma_{0}(T)\right\}
$$

and $\sigma(T)=\sigma_{w}(T)$.

Proof of Theorem 2.7. For given $\varepsilon>0$, set

$$
\Gamma_{1}=\left\{\lambda \in \sigma_{0}(T): \operatorname{dist}\left(\lambda, \partial \rho_{s-F}(T)\right) \geq \frac{\varepsilon}{2}\right\}
$$

Then $\Gamma_{1}$ is a finite, clopen subset of $\sigma(T)$. Set $\Gamma_{2}=\sigma(T) \backslash \Gamma_{1}$. By Corollary 3.7, $T$ admits the following representation

$$
T=\left[\begin{array}{cc}
A & C \\
0 & B
\end{array}\right] \quad \begin{gathered}
\mathscr{H}\left(\Gamma_{1} ; T\right) \\
\mathscr{H}\left(\Gamma_{1} ; T\right)^{\perp},
\end{gathered}
$$

where $\sigma(A)=\Gamma_{1}$ and $\sigma(B)=\Gamma_{2}$. Then one can verify that

$$
\max \left\{\operatorname{dist}\left[\lambda, \partial \rho_{s-F}(B)\right]: \lambda \in \sigma_{0}(B)\right\}<\frac{\varepsilon}{2} .
$$

Then, by Lemma 7.3 , there exists $K_{1} \in \mathscr{K}(\mathscr{H})$ with $\left\|K_{1}\right\|<\varepsilon / 2$ such that

$$
T+K_{1}=\left[\begin{array}{cc}
A & C \\
0 & B
\end{array}\right] \begin{gathered}
\mathscr{H}\left(\Gamma_{1} ; T\right) \\
\mathscr{H}\left(\Gamma_{1} ; T\right)^{\perp},
\end{gathered}
$$

where $\bar{B}$ is a compact perturbation of $B$ and $\sigma(\bar{B})=\sigma_{w}(\bar{B})$. It is obvious that $\sigma(\bar{B}) \subset \sigma(B)=\Gamma_{2}$. Hence $\sigma(A) \cap \sigma(\bar{B})=\emptyset$. By Theorem 7.2, there exists a compact $\overline{K_{2}}$ on $\mathscr{H}\left(\Gamma_{1} ; T\right)^{\perp}$ with $\left\|\overline{K_{2}}\right\|<\varepsilon / 4$ such that $\left[\sigma(\widehat{B}) \backslash \sigma_{1}(\widehat{B})\right] \subset$ $\left[\operatorname{conv} \sigma_{1}(\widehat{B})\right]^{\circ}$, where $\widehat{B}=\bar{B}+\overline{K_{2}}$. 
Since $A$ is an operator on $\mathscr{H}\left(\Gamma_{1} ; T\right)$ and $\operatorname{dim} \mathscr{H}\left(\Gamma_{1} ; T\right)<\infty$, there exists a compact $\overline{K_{3}}$ on $\mathscr{H}\left(\Gamma_{1} ; T\right)$ with $\left\|\overline{K_{3}}\right\|<\varepsilon / 2$ such that $\sigma(\widehat{A})=\sigma_{1}(\widehat{A})$, where $\widehat{A}=A+\overline{K_{3}}$. By the upper semi-continuity of spectrum, we may also assume that $\sigma(\widehat{A}) \cap \sigma(\widehat{B})=\emptyset$.

Set

$$
K=K_{1}+\left[\begin{array}{cc}
\overline{K_{3}} & 0 \\
0 & \overline{K_{2}}
\end{array}\right] \quad \begin{aligned}
& \mathscr{H}\left(\Gamma_{1} ; T\right) \\
& \mathscr{H}\left(\Gamma_{1} ; T\right)^{\perp}
\end{aligned} .
$$

Then $K \in \mathscr{K}(\mathscr{H}),\|K\|<\varepsilon$ and

$$
T+K=\left[\begin{array}{cc}
\widehat{A} & C \\
0 & \widehat{B}
\end{array}\right] \quad \begin{aligned}
& \mathscr{H}\left(\Gamma_{1} ; T\right) \\
& \mathscr{H}\left(\Gamma_{1} ; T\right)^{\perp}
\end{aligned}
$$

It is easy to verify that $\left[\sigma(T+K) \backslash \sigma_{1}(T+K)\right] \subset\left[\operatorname{conv} \sigma_{1}(T+K)\right]^{\circ}$. Thus $T+K \in \mathcal{S}(\mathscr{T})^{\circ}$ and we conclude the proof.

It follows from Theorem 2.6 that each operator $T \in \mathscr{B}(\mathscr{H})$ is a compact perturbation of some transloid (also normaloid) operator. The following result shows that not every operator is a compact perturbation of some p-normaloid operator.

Proposition 7.4. Let $T \in \mathscr{B}(\mathscr{H})$. Suppose that $\sigma(T)=\{0\}$ and $T \notin \mathscr{K}(\mathscr{H})$. Then $T+K \notin \mathcal{S}(\mathscr{P})$ for all $K \in \mathscr{K}(\mathscr{H})$.

Proof. If $A \sim T$, then it is obvious that $\sigma(A)=\{0\}$ and $A \notin \mathscr{K}(\mathscr{H})$. Thus it suffices to prove that $T+K \notin \mathscr{P}$ for all $K \in \mathscr{K}(\mathscr{H})$. For a proof by contrary, we assume that $T+K \in \mathscr{P}$ for some $K \in \mathscr{K}(\mathscr{H})$. Denote $T^{\prime}=T+K$. Then $p\left(T^{\prime}\right)$ is normaloid for each polynomial $p(\lambda)$.

Obviously $\sigma\left(T^{\prime}\right)$ consists of at most denumerable points and has no nonzero limit point. Then each continuous function on $\sigma\left(T^{\prime}\right)$ can be uniformly approximated by polynomials. By Corollary 4.2, it follows that $f\left(T^{\prime}\right)$ is normaloid for all $f \in \operatorname{Hol}\left(\sigma\left(T^{\prime}\right)\right)$, that is, $T^{\prime} \in \mathscr{V}$. Using a classical result of von Neumann (see [13] or [4, Theorem 9.9]]), we can deduce that $T^{\prime}$ is normal. Hence $T$ has the form "normal plus compact". Note that $T$ is quasinilpotent, then, by the well-known B-D-F Theorem, one can deduce that $T \in \mathscr{K}(\mathscr{H})$, a contradiction.

\section{References}

[1] C. Apostol, L. A. Fialkow, D. A. Herrero, and D. Voiculescu, Approximation of Hilbert space operators. Vol. II, Research Notes in Mathematics, 102. Pitman (Advanced Publishing Program), Boston, MA, 1984.

[2] C. Apostol and B. B. Morrel, On uniform approximation of operators by simple models, Indiana Univ. Math. J. 26 (1977), no. 3, 427-442.

[3] J. B. Conway, A Course in Functional Analysis, Second edition.Graduate Texts in Mathematics, 96. Springer-Verlag, New York, 1990.

[4] _ The Theory of Subnormal Operators, Mathematical Surveys and Monographs, 36. American Mathematical Society, Providence, RI, 1991. 
[5] F. Gilfeather, Norm conditions on resolvents of similarities of Hilbert space operators and applications to direct sums and integrals of operators, Proc. Amer. Math. Soc. 68 (1978), no. 1, 44-48.

[6] P. R. Halmos, A Hilbert Space Problem Book, Second edition.Graduate Texts in Mathematics, 19. Encyclopedia of Mathematics and its Applications, 17. Springer-Verlag, New York-Berlin, 1982.

[7] D. A. Herrero, A Rota universal model for operators with multiply connected spectrum, Rev. Roumaine Math. Pures Appl. 21 (1976), no. 1, 15-23.

[8] _ Approximation of Hilbert Space Operators. Vol. 1, Second edition.Pitman Research Notes in Mathematics Series, 224. Longman Scientific \& Technical, Harlow; copublished in the United States with John Wiley \& Sons, Inc., New York, 1989.

[9] C. L. Jiang and Z. Y. Wang, Structure of Hilbert Space Operators, World Scientific Publishing Co. Pte. Ltd., Hackensack, NJ, 2006.

[10] W. Y. Lee, Lecture Notes on Operator Theory, Seoul National University, Seoul, 2010.

[11] H. Radjavi and P. Rosenthal, Invariant Subspaces, Second edition.Dover Publications, Inc., Mineola, NY, 2003.

[12] G.-C. Rota, On models for linear operators, Comm. Pure Appl. Math. 13 (1960), 469472 .

[13] J. von Neumann, Eine Spektraltheorie für allgemeine Operatoren eines unitären Raumes, Math. Nachr. 4 (1951), 258-281.

[14] A. Wintner, Zur theorie der beschränkten bilinearformen, Math. Z. 30 (1929), no. 1, $228-281$.

Sen ZHu

Department of Mathematics

JILIN UNIVERSITY

Changchun 130012, P. R. China

E-mail address: zhusen@jlu.edu.cn

Chun Guang Li

INSTITUTE OF MATHEMATICS

JILIN UNIVERSITY

Changchun 130012, P. R. China

E-mail address: licg09@mails.jlu.edu.cn 